\title{
Phronesis
}

\section{Quels discours pour quel développement professionnel ?}

\section{Kristine Balslev et Alexandre Buysse}

Volume 5, numéro 3-4, 2016

Quels discours pour quel développement professionnel ?

URI : https://id.erudit.org/iderudit/1039081ar

DOI : https://doi.org/10.7202/1039081ar

Aller au sommaire du numéro

Éditeur(s)

Université de Sherbrooke

Champ social éditions

ISSN

1925-4873 (numérique)

Découvrir la revue

Citer ce document

Balslev, K. \& Buysse, A. (2016). Quels discours pour quel développement professionnel ? Phronesis, 5(3-4), 1-4. https://doi.org/10.7202/1039081ar d'utilisation que vous pouvez consulter en ligne.

https://apropos.erudit.org/fr/usagers/politique-dutilisation/ 


\section{INTRODUCTION}

Numéro coordonné par:

\section{Kristine Balslev,}

Maître d'enseignement et de recherche, FPSE, Université de Genève,

\section{et}

\section{Alexandre Buysse,}

Professeur agrégé,

FSE, Université Laval

\section{Quels discours pour quel développe- ment professionnel?}




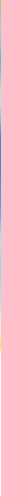

Dans les formations aux métiers éducatifs, l'activité professionnelle, souvent sous forme de stage, est au cœur de nombreux dispositifs. Dans la perspective des formations en alternance intégrative (Vanhulle, Merhan et Ronveaux, 2007 ; Vanhulle, 2012), les professionnels en formation sont amenés à produire des discours sur cette activité en vue, notamment, de développer leur réflexivité, de construire des savoirs professionnels pour, au final, développer un agir professionnel. Dans ces formations, un lien fort entre le retour sur l'action et l'action elle-même est posé, l'hypothèse de base étant que le discours a un impact sur les pratiques. Cette hypothèse n'est pas propre à la formation, elle est aussi, à certaines conditions, valable pour les discours des professionnels confrontés à leur pratique.

Les dispositifs invitant à intégrer savoirs théoriques et expériences professionnelles, constituent des lieux de production de discours professionnels qui représentent des espaces de réflexion amenant à la consignation du savoir, la construction de connaissances, la confrontation entre des savoirs et des expériences concrètes (Rabatel et Blanc, 2011). Ajoutons à cela l'activité réflexive du futur professionnel prônée, sinon exigée dans ces formations, passant par des procédures de discussion et de négociation (Vanhulle, 2008). Celle-ci implique, d'une part, une importante démarche de conceptualisation et de théorisation de la pratique et, d'autre part, un processus de positionnement permettant de discuter des valeurs et de l'agir professionnel et, plus généralement, de donner sens à son activité.

Ces discours se construisent en interaction et ont pour vocation d'être porteuses de médiations permettant à l'apprenant d'intérioriser et d'intégrer différents savoirs et processus (Buysse et Renaulaud, 2012). Mais ces discours sont également le théâtre d'autres phénomènes, tels la reconnaissance (Jorro, 2011 ; Vanhulle, 2009), la construction identitaire, le positionnement de soi (Rabatel et Blanc, 2011), I'adhésion progressive à une forme socioculturelle de pensée (Buysse, 2012) ou encore la présentation d'un point de vue (Rabatel, 2005). Se pose dès lors la question de l'effet des dispositifs et des formes interactives ou genres textuels sur le(s) développement(s) professionnel(s).

Ainsi, c'est la fonction du langage en usage dans la formation ou la profession, en lien avec des situations de stage ou de travail, qui est au cœur de ce numéro de Phronesis. Quels éléments du discours oral ou écrit sont à prendre en compte pour saisir le développement professionnel ? S'agissant de discours de formation et de discours professionnels, visant l'appropriation de savoirs, comment ces savoirs sont-ils reconfigurés par les discours? Comment s'arriment des phénomènes observables dans le discours avec des phénomènes intrapsychiques et invisibles ? À quelle formation forment les dispositifs ? Telles sont les questions abordées par ce numéro, dont la richesse réside dans la diversité des formes de discours, des dispositifs et des phénomènes étudiés.

Les discours oraux ou écrits étudiés dans ce numéro proviennent en partie de pratiques ordinaires et authentiques de formation : entretiens tripartites de stage (Dobrowolska et al.), interactions pendant le déroulement même du travail (Durand), mémoires professionnels (Rabatel), interactions lors d'activités de formation (Gagnon et Laurens), récits d'expériences sous la forme de correspondance épistolaire (Geffard et Dubois), échanges sur un forum de coélaboration du savoir (Boutin, Hamel \& Gouin), écrits et entretiens réflexifs (Buysse). Une autre partie provient de dispositifs de recherche : entretiens de recherche (Nizet) et entretiens en autoconfrontation (Cicurel ; Pham Quang) ; ou encore d'un dispositif de validation des acquis de l'expérience (Rémery). Il est intéressant de noter que, même si les recherches ne visent pas toutes directement le développement professionnel, les auteurs mettent en évidence un effet de la production de discours sur l'agir professionnel. En effet, les chercheurs suggèrent ou montrent, un impact de ces interactions sur - entre autres - la réflexivité, les actions, le positionnement, ou encore la reconnaissance des apprentissages par les professionnels ou les formés.

La proximité ou la distance entre discours et pratique professionnelle caractérise également les données étudiées dans ce numéro. En effet, certains des discours analysés sont produits en forte proximité avec l'activité professionnelle, comme les 
interactions formateur-formé pendant le déroulement même de l'activité (Durand) ou encore les interactions provenant d'une confrontation directe avec la pratique professionnelle filmée (Cicurel; Pham Quang). D'autres discours se situent dans un empan temporel proche de la pratique professionnelle sous forme de stage : interactions formatives, entretiens de recherche, ou écritures de récits ou de mémoires ayant lieu pendant la période de formation en alternance (Boutin et al. ; Dobrowolska et al.; Gagnon \& Laurens; Geffard et Dubois ; Nizet ; Rabatel). Enfin, d'autres discours s'appuient sur l'expérience professionnelle accumulée sur une période longue (Rémery).

Plusieurs auteurs estiment que les discours révèlent ou engendrent des processus intrapsychiques chez la personne en formation. Ainsi plusieurs auteurs traitent de la réflexivité (Boutin, Hamel et Gouin ; Buysse ; Dobrowolska et al.; Cicurel, Gagnon \& Laurens ; Geffard et Dubois ; Nizet ; Rabatel) qui constitue incontestablement un sujet phare de ce numéro. Ce n'est guère étonnant : la réflexivité fait souvent partie du contexte, du moins dans la formation des enseignants où cet élément est fortement sollicité. D'après Vanhulle (2008), la réflexivité s'élabore conjointement avec des formes de langage, par conséquent ses indices peuvent être observés et identifiés à travers l'analyse de discours. Dans la même veine, Cicurel montre ce que les verbalisations disent sur la pensée enseignante en lien avec l'action enseignante, et Buysse s'interroge sur le développement qui en résulte.

Une variété d'outils linguistiques sont mis en exergue dans ce numéro de Phronesis, tels le positionnement (Dobrowolska et al.; Rabatel), la prise et l'offre de place pendant les activités professionnelles du stagiaire (Durand), le cadrage et sa transformation (Rémery), la référentialisation (Quang Pham; Rabatel; Rémery) et le point de vue adopté dans le discours (Rabatel).

L'originalité de ce numéro réside également dans la place accordée aux savoirs; dans plusieurs contributions, il est question d'intégrations de différents types de savoirs (Buysse ; Dobrowolska et al.), de reconfiguration des savoirs (Nizet) et de circulation des savoirs (Gagnon et Laurens). La conception des savoirs professionnels élaborée par Vanhulle (2008; 2009 ; Vanhulle, Buysse \& Balslev, 2012), a inspiré bon nombre de ces auteurs et est particulièrement appropriée pour une compréhension du développement professionnel en tant que processus en partie langagier, intégrant de la réflexivité et des savoirs.

Plusieurs auteurs de ce numéro mettent en évidence également les liens forts unissant la formation et l'insertion dans une communauté de pratique ou d'apprentissage (Boutin et al.). Buysse met l'accent sur cet aspect en considérant que ces communautés se situent au sein d'activités culturelles, dotées de formes de pensée et de discours qui leur sont propres et que l'apprenant s'approprie progressivement. Ainsi se former consisterait à participer à différentes activités (académiques, d'enseignement et réflexives).

L'étude de ces différents phénomènes met en évidence la complexité du développement professionnel. Celui-ci prend comme terreau les savoirs proposés par les formations, les référentiels et les prescriptions de différentes formations, les actions professionnelles vécues ou projetées; se réalise dans des discours qui sont configurés par différentes communautés de pratiques et d'apprenants en fonction des finalités qu'elles visent. Le développement d'un agir professionnel dépend de positionnements pendant le déroulement même de cet agir et dans le retour réflexif.

Des mots, encore des mots, toujours des mots pour se former, prendre conscience, devenir réflexif ? Plusieurs auteurs nous invitent à nous questionner sur l'importance accordée au verbal. Cicurel, qui s'appuyant sur Schlanger (2010) affirme que le matériau mental n'est pas fait que de mots, mais que ce matériau est le seul à même de transmettre des idées. En affirmant cela, elle relativise la fonction des verbalisations, tout en montrant leurs apports. Dans le même sens, Buysse suggère que, même « si le langage joue un rôle important, il n'est probablement pas la source principale des processus qui sont mobilisés dans la formation à une activité socioculturelle.»

En résumé, ce numéro met en évidence les indices visibles dans les discours, permettant de comprendre une part du développement et de l'agir professionnels, tout en montrant que le développement ne se réduit pas à des éléments interactionnels et discursifs.

\section{Références bibliographiques}

Buysse, A. (2012). La formation des enseignants en tant que transmission d'une forme socioculturelle. Phronesis (1), 4, 4-20. Buysse, A. et Renaulaud, C. (2012). Enseignants du secondaire et bilan de compétences : quelles appropriations ? Formation et pratiques d'enseignement en questions, 15, 73-96.

Jorro, A. (2011). Evaluation de l'expérience et enjeux de reconnaissance professionnelle. Les Sciences de l'éducation. Pour l'Ere nouvelle., 44(2), 69-82. 
Rabatel, A. (2005). Le point de vue, une catégorie transversale. Le français aujourd'hui, 151, 57-68.

Rabatel, A. et Blanc, N. (2011). Construire une expertise dans et par les discours professionnels. Lidil, 43, 5-10.

Schlanger, J. (2010). Le jeu des idées. Paris : Hermann philosophie.

Vanhulle, S. (2008). Réflexivité des enseignants. In A. Van Zanten (dir.), Dictionnaire de l'éducation (pp. 259-261). Paris, France : Presses Universitaires de France.

Vanhulle, S. (2009). Quand la reconnaissance donne forme aux savoirs professionnels. In A. Jorro (dir.), La reconnaissance professionnelle en éducation. Evaluer, valoriser, légitimer (pp. 61-76). Ottawa, ON: Presses Universitaires d'Ottawa.

Vanhulle, S., Merhan, F., \& Ronveaux, C. (2007). Introduction: du principe d'alternance aux alternances en formation des adultes et des enseignants: un état de la question. In F. Merhan, C. Ronveaux \& S. Vanhulle (Eds.), Alternances en formation (pp. 5-45). Bruxelles: de Boeck.

Vanhulle, S. (2012). Quand l'activité professionnel s'invite dans la formation académique: le cas des stages en enseignement. In E. Bourgeois et M. Durand (dir.), Apprendre au travail (pp. 165-175). Paris, France: Presses Universitaires de France.

Vanhulle, S., Balslev, K., \& Buysse, A. (2012). Comprendre les processus discursifs de la construction de savoirs professionnels et leurs effets régulateurs en termes de développement. In J. Clénet, P. Maubant \& D. Poisson (Eds.), Formations et professionalisations: à l'épreuve de la complexité. (pp. 117-150). Paris: L'Harmattan. 